\title{
Memória de trabalho viso-espacial em crianças de 7 a 12 anos $^{1}$
}

\author{
Ederaldo José Lopes \\ Renata Ferrarez Fernandes Lopes \\ Universidade Federal de Uberlândia \\ Cesar Alexis Galera \\ Universidade de São Paulo - Ribeirão Preto
}

\begin{abstract}
Resumo
O objetivo deste trabalho foi avaliar os mecanismos de processamento da informação viso-espacial em crianças. Setenta e oito crianças participaram do experimento em que foram manipulados os fatores idade, posição espacial, similaridade visual e cor dos estímulos memorizados. Os resultados mostraram que todos os fatores principais alcançaram significância estatística. As crianças mais velhas tiveram uma freqüência de acertos maior que as crianças mais novas. Os estímulos dos conjuntos com similaridade baixa foram mais bem recordados que os estímulos com similaridade alta. A taxa de recordação foi melhor nas provas em que as letras de um conjunto foram todas apresentadas com a mesma cor, assim como a porcentagem de respostas corretas variou de forma significativa em função da posição espacial dos estímulos. Os resultados foram interpretados de acordo com modelos que enfatizam aspectos do desenvolvimento de estratégias cognitivas ao longo do desenvolvimento humano, especialmente o modelo de memória de trabalho.
\end{abstract}

Palavras-chave: memória de trabalho; memória viso-espacial; memória fonológica; desenvolvimento humano

\begin{abstract}
Visuo-spatial working memory in 7-12 year old children. This study aimed to evaluate the mechanisms of visual-spatial memory in children. Seventy eight children took part in an experiment with four factors: children's age, stimuli spatial position, stimuli visual similarity, stimuli set color. The results have shown that all main factors are statistically meaningful. The oldest children presented a better performance than the youngest ones. Stimuli set formed by low similarity letters were better recollected than the stimuli set formed by high similarity letters. The recall of the spatial position of letters was better in trials where the letters of a set were presented in the same color. The percentage of correct recall changed meaningfully as a function of the spatial position in which the target had been presented. The results were interpreted according to models that emphasize aspects of development of cognitive strategies along with the human development, especially the working memory model.
\end{abstract}

Keywords: working memory; visuo-spatial memory; phonological memory; human development

A preocupação em determinar a forma pela qual a informação visual é representada na memória tem ensejado inúmeras discussões e proposições de modelos de processamento da informação, bem como de modelos de armazenamento e recuperação da informação na memória. Um dos modelos mais promissores é aquele denominado working memory (memória de trabalho), proposto por Baddeley e Hitch (1974; para revisões, ver Baddeley, 1986, 1992, 1994, 2000). Esse modelo tem focalizado o funcionamento cognitivo, incluindo atividades como pensamento, solução de problemas e memória, bem como atividades relacionadas ao processamento da linguagem, tais como compreensão e leitura (Stoltzfus, Hasher, \& Zacks, 1996). O modelo de memória de trabalho ampliou e, em certo sentido, substituiu os conceitos anteriores formulados no modelo modal de Atkinson e Shiffrin (1968), mostrando que a memória não é um sistema unitário, mas múltiplo. Da forma como foi proposto originalmente, o modelo de memória de trabalho envolvia um executivo central, que atua como um sistema atencional; um esboço viso-espacial, que trabalha como um sistema de armazenamento visual e/ou espacial e um laço fonológico, que trabalha como um sistema de recitação. Mais recentemente, o modelo passou a contar com um quarto componente, denominado buffer episódico, um sistema temporário de capacidade limitada cuja função é unir a informação dos sistemas escravos à informação da memória de longo prazo, formando uma representação episódica unitária (Baddeley, 2000). 
O laço fonológico é a parte mais estudada do sistema de memória de trabalho. As evidências experimentais sugerem que o armazenamento e a manipulação do material verbal são realizados por meio de dois subcomponentes: um breve armazenador, baseado na fala, que mantém a informação na memória por um curto espaço de tempo e um processo de controle articulatório que mantém a informação na memória via um mecanismo de recitação interna (reverberação), que consiste na repetição interna (subvocal) da informação visual ou auditiva. O material verbal apresentado auditivamente tem acesso obrigatório ao armazenador fonológico, que é relativamente passivo. O mecanismo de recitação ajuda a manter a informação armazenada e recodifica em termos verbais o material apresentado visualmente (Baddeley, 1990; Brandimonte \& Gerbino, 1996). O laço fonológico é limitado temporalmente, podendo conter tantos itens quantos puderem ser recitados no intervalo de 1,5 a 2 segundos. Portanto, está ligado à taxa de articulação. O funcionamento desse subsistema explica os fenômenos observados nos estudos de memória verbal a curto prazo, tais como os efeitos do tamanho de palavra, supressão articulatória e efeitos da similaridade fonológica (Baddeley, 1986). O rascunho viso-espacial é responsável pelo armazenamento e manipulação da informação visual e/ou espacial. Ele pode ser considerado como uma interface entre a informação visual e a informação espacial, informações estas que podem ser acessadas via órgãos de sentido ou via memória de longo prazo (Baddeley, 2002). Embora os estudos desse componente tenham aumentado de forma considerável nos últimos anos, ainda permanecem em aberto muitas questões, principalmente aquelas concernentes ao mecanismo de recitação desse sistema (Baddeley, 1986).

As diferenças de processamento entre a informação verbal/fonológica e viso-espacial são bem documentadas na literatura (Logie, 1995). Todavia, essas diferenças não são muito claras, pois existem evidências de que tendemos a utilizar rótulos verbais mesmo para estímulos visuais para os quais não dispomos de nomes (Bahrick \& Boucher, 1968; Brandimonte \& Gerbino, 1996; Broadbent \& Broadbent, 1981). Além disso, vários estudos comportamentais têm sugerido a existência de sistemas separados para o armazenamento da informação visual e espacial (Della Sala, Gray, Baddeley, Allamano, \& Wilson, 1999; Hecker \& Mapperson, 1997; Logie \& Pearson, 1997; Pickering, Gathercole, \& Peaken, 1998; Postma \& De Haan, 1996; Quinn \& McConnell, 1996). Estes estudos confirmam evidências neuropsicológicas que apontam para a separação entre o processamento da informação sobre o que é o objeto e sobre sua localização espacial, identificadas com as vias de processamento ventral e dorsal (Milner \& Goodale, 1995).

Estudos com crianças são utilizados para se demonstrar como e quando se desenvolvem os componentes verbais e viso-espaciais da memória de trabalho. Alguns desses estudos (Gathercole \& Hitch, 1993; Hitch, Halliday, Dodd, \& Littler, 1989; Hitch, Halliday, \& Littler, 1989; Hitch, Halliday, Schaafstal, \& Schraagen, 1988; Hitch, Woodin, \& Baker, 1989; ver também Walker, Hitch, Doyle, \& Porter, 1994) mostraram que o processo de recitação articulatória se desenvolve com a idade, embora as idades em que a recitação foi observada variem com as situações estudadas.

O efeito de tamanho de palavra tem sido encontrado em crianças de 4 anos quando estímulos verbais são apresentados de maneira auditiva (Hulme, Thomson, Muir, \& Lawrence, 1984; Hulme \& Tordoff, 1989). Em contraste, com apresentação visual (desenhos de objetos), os efeitos de tamanho de palavra não aparecem antes de 7 ou 8 anos de idade. Crianças mais velhas (pelo menos de 8 anos em diante) usam espontaneamente a recitação articulatória quando a apresentação dos estímulos é auditiva ou visual. Incidentalmente, é interessante observar que esses resultados convergem com aqueles de Flavell (Flavell \& Wellman, 1977) e trabalhos de outros pesquisadores sobre recitação espontânea em tarefas de memória. Hitch et al. (1988) sugeriram que a codificação verbal não substitui meramente a codificação visual, mas que o desenvolvimento consiste de uma multiplicação do número possível de sistemas de codificação. Mudanças desenvolvimentais em tarefas que exigem o rascunho viso-espacial também têm sido investigadas. Schumann-Hengsteler, Demmel e Seitz (1992) mostraram que há pouca mudança qualitativa entre crianças de 5 e 10 anos na habilidade para desempenhar tarefas que exigem o rascunho viso-espacial quando tais tarefas não variam em termos de complexidade (ver também, Hitch et al., 1988; Hitch et al., 1989). Embora pelo menos um estudo (Mandler, Seegmiller, \& Day, 1977) não tenha mostrado diferenças claras entre crianças e adultos, outros estudos têm mostrado padrões desenvolvimentais em crianças (Park \& James, 1983) e mudanças entre infância e idade adulta (von Wright, Gebhard, \& Karttunen, 1975). Outros ainda mostraram que os adultos tiveram um desempenho melhor que as pessoas idosas em tarefas de memória para localização espacial (e.g., Light \& Zelinski, 1983; Park, Puglisi, \& Lutz, 1982; ver também Pezdek, 1983). Schulmann-Hengsteler (1992), trabalhando com crianças de 4 a 10 anos, em um experimento em que deveriam olhar para um display por 4 segundos e depois reconstruí-lo, encontrou uma melhora para a memorização dos itens com a idade, bem como para a associação item-localização.

No presente estudo, pretendemos avaliar o processamento da informação viso-espacial em crianças de seis faixas etárias que cobrem o início do processo de leitura. Para esse fim, empregamos uma tarefa de recordação da localização espacial utilizando letras como estímulos. De acordo com a suposição de que as crianças utilizam inicialmente um processo de armazenamento e recitação baseado em características visuais, devemos supor que o desempenho de crianças mais jovens será mais prejudicado por fatores visuais que afetem a tarefa de recordação, tal como a similaridade visual ou a diferença de cor entre os estímulos (Walker, Hitch, \& Duroe, 1993; Walker et al., 1994).

Walker et al. (1993, Exp. 3) manipularam a similaridade visual de letras fazendo modificações na aparência delas, de 
modo a exacerbar características já divididas por elas ou introduzindo características que serviram de base para a medida de similaridade. Os participantes (adultos) viram uma lista de 4 letras e, após um curto intervalo, apresentou-se-lhes um estímulo teste numa localização espacial neutra, situada abaixo da lista memorizada. A tarefa do participante consistiu em indicar em que localização espacial da lista o estímulo teste tinha sido apresentado. A percentagem de respostas corretas foi afetada pela similaridade visual, com uma vantagem das provas visualmente dissimilares entre si, relativamente às provas visualmente similares. Esse efeito da similaridade foi interpretado como indicativo de que a memória de curta duração para a localização espacial é mediada por um armazenador visual que não está restrito aos itens apresentados mais recentemente. Num outro estudo, Walker et al. (1994, Exp. 1) manipularam a similaridade visual de letras de duas formas: variando a forma e a cor dos estímulos. Sendo realizado com crianças, a tarefa consistiu em indicar a posição espacial em que uma das letras tinha sido apresentada. O desempenho das crianças piorou na condição de similaridade alta, sugerindo a participação de um armazenador visual. O Experimento 2 comparou a memória de crianças de 5-7 anos para a cor da forma ou sua localização espacial. Embora tenha havido uma melhora na memória para localização espacial, ela se restringiu aos itens pré-recência. Não houve efeito da idade em relação à recordação de cor.

Finalmente, embora a literatura em geral seja controversa quanto aos efeitos da idade sobre a codificação e localização espacial de objetos (McCormack, 1982; Park \& James, 1983; Pezdek, 1983), devemos supor que o desempenho melhore com a idade, uma vez que as crianças mais velhas podem empregar, além de elementos viso-espaciais, mecanismos mais eficazes de recitação baseados nos nomes das letras, comparativamente às crianças mais novas (Pickering et al., 1998).

\section{Método}

\section{Participantes}

Ao todo, 78 crianças participaram deste estudo, divididas igualmente entre as idades de 7, 8, 9, 10, 11 e 12 anos, das quais 39 eram meninas e 39 eram meninos. À época do experimento, todos eram estudantes de uma escola municipal de uma cidade da região de Ribeirão Preto, SP.

\section{Estímulos e material}

Foram usados 24 conjuntos compostos por 3 letras. Seis conjuntos foram formados por letras pouco similares ente si (GRF-RGF-BTQ-TQB-LCP-CPL); todas as letras de um conjunto foram apresentadas numa mesma cor, que poderia ser vermelha, verde ou azul. Outros 6 conjuntos foram formados pelas mesmas letras anteriores, mantendo o mesmo grau de similaridade, porém cada letra do conjunto foi apresentada em uma das cores, escolhida de forma aleatória. Seis outros conjuntos foram formados por letras com similaridade alta (GCQ-CQG-LTF-FTL-RBP-BPR), de maneira que todas as letras de um conjunto foram apresentadas na mesma cor. Final- mente, os últimos seis conjuntos foram formados pelas letras com grau de similaridade alta, apresentadas em cores diferentes, escolhidas aleatoriamente. A similaridade entre as letras foi estimada com base em métodos clássicos da literatura que permitem estimar a similaridade entre estímulos a partir do número de componentes ou características que têm em comum (Podgorny \& Garner, 1979; Van Ness \& Bouma, 1980).

Os estímulos foram apresentados na tela colorida (resolução de 800 x 600 pixels) de um micro-computador IBM-PC, 386DX, controlados por um programa construído para este fim em linguagem $C$. As letras memorizadas foram apresentadas dentro de três quadrados de $4 \mathrm{~cm}^{2}$, dispostos numa linha horizontal no centro da tela, com 2,5 cm de distância entre cada quadrado. $\mathrm{O}$ estímulo teste foi apresentado sempre na cor preta, num quadrado adicional situado a $4 \mathrm{~cm}$ abaixo da linha horizontal. O tamanho aproximado das letras foi 1,2 x 1,2 $\mathrm{cm}$. Os estímulos foram apresentados a uma distância de aproximadamente $50 \mathrm{~cm}$ do participante. A Figura 1 mostra exemplos dos estímulos utilizados no experimento.
(A)
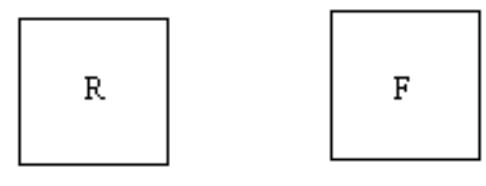

$\mathrm{R}$

(B)
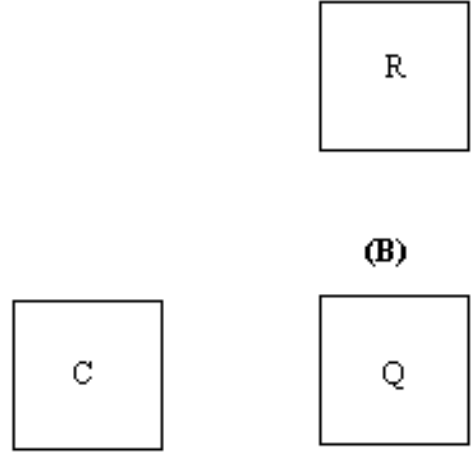

G

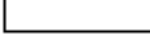

$\mathrm{C}$

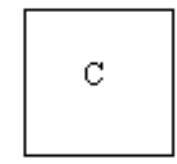

Figura 1. Delineamento experimental: $(\mathrm{A})=$ conjunto com nível de similaridade baixa, com letras apresentadas na mesma cor; (B) = conjunto com nível de similaridade alta, com as letras $\mathrm{C}, \mathrm{Q}$ e $\mathrm{G}$ apresentadas em cores diferentes.

\section{Procedimento}

As letras foram apresentadas em sequiências aleatórias nas três diferentes posições. Cada prova tinha início quando o experimentador pressionava a tecla de espaço do teclado do computador. Um segundo depois, a primeira letra era apresentada em um dos quadrados da parte superior da tela do computador. A primeira letra permanecia exposta por 1,5 segundos. Quando ela desaparecia da tela, seguia-se um inter- 
valo de 0,5 segundo, após o qual aparecia a outra letra, e assim por diante, até a terceira letra. Depois que a terceira letra desaparecia, havia um intervalo de 1,5 segundos, após o qual apresentava-se o estímulo teste. Este permanecia exposto até que uma resposta fosse dada. O participante identificava, com o dedo indicador na tela do computador, o quadrado no qual o estímulo teste tinha sido apresentado. O experimentador registrava a resposta do participante, pressionando uma das teclas (E para esquerda, C para centro e D para direita). O programa registrava a resposta como certa ou errada. A partir daí, iniciava-se uma nova prova com a mesma seqüência de passos.

Os participantes realizaram 24 provas, sendo 6 com similaridade baixa e cor igual, 6 com similaridade baixa e cor diferente, 6 com similaridade alta e cor igual, e 6 com similaridade alta e cor diferente. Em cada bloco de 6 provas, em duas o estímulo teste era apresentado na posição esquerda; em outras duas, na posição central e, nas duas restantes, na posição direita. A ordem de apresentação das provas foi aleatorizada. Aplicou-se um planejamento fatorial 6x3x2x2. Dos quatro fatores experimentais, um fator foi intersujeitos (idade com 6 níveis) e três fatores intra-sujeitos: posição espacial, (esquerda, centro e direita), similaridade visual (alta e baixa) e cor dos estímulos memorizados (igual e diferente). As percentagens individuais de respostas corretas foram submetidas a uma análise de variância (ANOVA) mista, utilizando-se o programa SPSS (Kinnear \& Gray, 1995).

\section{Resultados}

As percentagens médias das respostas corretas por fator experimental podem ser visualizadas na Tabela 1 .

Nos resultados da ANOVA, todos os fatores principais alcançaram significância estatística. Em termos gerais, as crianças mais velhas têm uma percentagem de acertos maior que as crianças mais novas, o que é confirmado pelo efeito significativo do fator idade $\left(F_{(5 ; 72)}=5,19 ; p<0,001\right)$. As posições dos estímulos nos conjuntos formados por letras com similaridade baixa são recordadas numa faixa de 7\% acima relativamente aos conjuntos formados por estímulos de similaridade alta, $\left(F_{(1 ; 72)}=25,27 ; p<0,001\right)$. A taxa de recordação é $4 \%$ melhor nas provas em que todas as letras do conjunto foram apresentadas na mesma cor $\left(F_{(1 ; 72)}=9,74 ; p<0,007\right)$. A percentagem de respostas corretas varia de forma significativa em função da posição espacial na qual foram apresentados os estímulos $\left(F_{(2 ; 144)}=11,7 ; p<0,001\right)$. Embora nenhuma interação tenha alcançado um nível de significância estatisticamente aceitável (em todos os resultados, $p>0,05$ ), pode-se observar na Tabela 1 que o efeito da similaridade tende a ser maior (9\%) nas crianças mais jovens do que nas crianças maiores (5\%). A mesma tendência ocorre com o uso da cor. O desempenho das crianças mais jovens foi mais prejudicado (5\%) do que o desempenho das crianças mais velhas (2\%) quando as letras foram apresentadas em cores diferentes. As comparações aos pares, usando o teste de Bonferroni, confirmam a vantagem geral das crianças na faixa de 11-12 anos em relação às crianças de 7-8 anos: foram encontradas diferenças significativas entre as idades de 7 e 11 anos (Diferença Média $(D M)=14,1 ;$ Erro Padrão $(E P)=3,87 ; p<0,01)$, 7 e 12 anos $(D M=16,9 ; E P=3,87 ; p<0,01)$, 8 e 12 anos $(D M=12,5$; $E P=3,87 ; p<0,05)$. Nenhuma outra diferença significativa foi encontrada para o fator idade (em todos os resultados, $p>$ $0,05)$. Para o fator similaridade, a $D M$ foi igual a 7,47 (EP = 1,48; $p<0,001$ ), confirmando a facilidade maior na localização do estímulo teste na condição de similaridade baixa. Para o fator cor, a $D M=4,27$ ( $E P=1,36$; $p<0,01)$ confirma a superioridade na localização espacial do estímulo teste quando este era da mesma cor que os estímulos memorizados. Finalmente, para o fator posição espacial, as comparações mostram as seguintes diferenças: entre as posições esquerda e direita $(D M=5,76 ; E P=1,84 ; p<0,01)$, confirmando a facilitação da localização de estímulos na posição direita; entre as posições central e direita, com vantagem para esta última $(D M=8,97$; $E P=1,76 ; p<0,001)$.

\section{Discussão}

As comparações post-hoc confirmam a melhora no desempenho das crianças nas duas últimas idades (11 e 12 anos). Além disso, todos os fatores visuais manipulados mostraram efeitos significativos sobre os participantes de todas as idades. Essa susceptibilidade às manipulações das características visuais dos estímulos indica que, de algum modo, todos os participantes realizaram a tarefa de localização espacial de letras levando em conta o componente viso-espacial da memória de trabalho. Esse efeito é semelhante àquele observado em crianças e adultos quando do uso da supressão articulatória, que tem como um de seus efeitos principais um aumento na sensibilidade às manipulações da similaridade visual dos estímulos (Walker et al., 1993; Walker et al., 1994). O desempenho dos participantes foi prejudicado pelo aumento da similaridade entre as letras. O efeito da similaridade visual, em torno de $7 \%$, sugere que as características visuais das letras, assim como de estímulos visuais sem nome, podem ser suficientes para o processo de localização e, pode-se supor, em termos do modelo de memória de trabalho, que o participante trabalha com o nível mais imediato disponível ao processamento, neste caso o nível visual (Galera \& Fuhs, 2003; Walker et al., 1993). A similaridade entre estímulos afeta o desempenho das crianças da mesma forma que afeta o desempenho de adultos, como na maioria dos estudos encontrados na literatura (e.g., Logie, 1995). Estudos com adultos têm mostrado, como nossos resultados com crianças mostram, que é mais fácil recordar a posição de estímulos mais diferentes entre si dos que de estímulos semelhantes. No estudo de Walker et al. (1993), a recordação da posição de letras pouco similares entre si foi aproximadamente $10 \%$ melhor do que de letras mais similares entre si. No estudo de Galera e Fuhs (2003), essa diferença foi de apenas 3\%. O efeito da similaridade entre as letras apresentadas tende a diminuir com o aumento da idade, suge- 
rindo que a informação visual pode estar sendo substituída por uma codificação verbal mais eficiente pelas crianças mais velhas. Dados da literatura (Hitch et al., 1988) mostraram esses mesmos efeitos em crianças muito novas no que tange à memória imediata para figuras desenhadas representando objetos do cotidiano, tais como animais, móveis, brinquedos, etc., bem como nos trabalhos feitos com adultos. Hitch et al. (1988) mostraram que as crianças de 5 anos de idade dependiam de uma memória ativa visual numa tarefa de recordação de desenhos de objetos cotidianos quando eles eram similares visualmente. No entanto, para as crianças de 10 anos de idade, esse efeito não foi encontrado. Em um estudo com adultos, Walker et al. (1993) mostraram efeitos deletérios da similaridade visual para padrões visuais de difícil nomeação (ou não-nomeáveis), ou mesmo letras que foram apresentadas numa forma um pouco diferente de seu padrão normal. Todavia, quando as letras apareciam na forma padronizada, o efeito da similaridade desaparecia.

A heterogeneidade (dissimilaridade) da cor das letras apresentadas como estímulos também têm um efeito deletério sobre o desempenho. A localização de letras apresentadas em cores iguais é recordada aproximadamente com uma vantagem de 3\% em relação à localização de letras apresentadas em cores diferentes. Mas aqui existe uma questão: se as cores tornam as letras mais diferentes entre si, isto é, aumentam a diferença entre as letras, por que a recordação das letras com cores diferentes é pior do que a recordação das letras com as mesmas cores, ou seja, mais semelhantes entre si? Walker et al. (1994) manipularam a similaridade baseada na cor dos estímulos memorizados e mostraram não haver diferenças significativas entre os estímulos similares e dissimilares. Eles consideraram esse resultado como uma evidência contrária à visão de que as crianças se basearam em códigos visuais para a recordação da localização espacial. Todavia, esse resultado é de difícil interpretação, pois a associação entre um item e sua localização não necessita logicamente da cor para que seja realizada corretamente. Em outra linha de explicação, os resultados provenientes do processamento da cor e da posição espacial podem estar refletindo o papel desempenhado pelos mecanismos atencionais na linha teórica de Treisman (e.g., Treisman, 1977; Treisman, 1988; Treisman \& Gelade, 1980). Ou seja, as dificuldades encontradas no processamento de itens de cores

Tabela 1

Percentagem média de acertos considerando as faixas etárias, os níveis de similaridade, as posições espaciais do estímulo teste e as cores das letras

\begin{tabular}{|c|c|c|c|c|c|c|c|c|c|c|c|c|}
\hline \multicolumn{13}{|c|}{ Posição do estímulo teste na condição cor igual } \\
\hline \multirow{2}{*}{$\begin{array}{l}\text { Idade } \\
\text { (anos) }\end{array}$} & \multicolumn{5}{|c|}{ Similaridade Alta } & \multicolumn{5}{|c|}{ Similaridade Baixa } & \multirow{2}{*}{$\begin{array}{l}\text { Média } \\
\text { Geral }\end{array}$} & \multirow{2}{*}{$\begin{array}{l}\text { Desvio- } \\
\text { Padrão }\end{array}$} \\
\hline & Esquerda & Centro & Direita & Média & $\begin{array}{l}\text { Desvio- } \\
\text { Padrão }\end{array}$ & Esquerda & Centro & Direita & Média & $\begin{array}{l}\text { Desvio- } \\
\text { Padrão }\end{array}$ & & \\
\hline 7 & 73 & 73 & 80 & 76 & 30 & 80 & 80 & 88 & 83 & 26 & 79 & 28 \\
\hline 8 & 76 & 73 & 92 & 81 & 25 & 80 & 80 & 92 & 85 & 26 & 83 & 25 \\
\hline 9 & 80 & 84 & 84 & 83 & 26 & 88 & 84 & 96 & 90 & 20 & 87 & 24 \\
\hline 10 & 84 & 80 & 92 & 86 & 23 & 88 & 84 & 96 & 90 & 20 & 88 & 22 \\
\hline 11 & 88 & 84 & 92 & 88 & 24 & 96 & 92 & 96 & 95 & 15 & 92 & 20 \\
\hline 12 & 92 & 82 & 92 & 91 & 19 & 96 & 92 & 100 & 96 & 13 & 94 & 17 \\
\hline Média & 82 & 80 & 89 & & & 88 & 85 & 94 & & & & \\
\hline \multirow[t]{2}{*}{$\begin{array}{l}\text { Desvio- } \\
\text { Padrão } \\
\end{array}$} & 26 & 24 & 23 & & & 22 & 24 & 15 & & & & \\
\hline & \multicolumn{12}{|c|}{ Posição do estímulo teste na condição cor diferente } \\
\hline 7 & 69 & 53 & 69 & 64 & 34 & 73 & 76 & 88 & 79 & 32 & 72 & 34 \\
\hline 8 & 73 & 61 & 76 & 71 & 36 & 84 & 80 & 88 & 85 & 26 & 78 & 32 \\
\hline 9 & 76 & 76 & 80 & 78 & 25 & 84 & 80 & 92 & 86 & 23 & 82 & 24 \\
\hline 10 & 80 & 80 & 88 & 83 & 26 & 88 & 80 & 92 & 87 & 22 & 85 & 24 \\
\hline 11 & 84 & 80 & 88 & 85 & 26 & 88 & 88 & 96 & 91 & 19 & 88 & 23 \\
\hline 12 & 88 & 84 & 88 & 87 & 22 & 96 & 92 & 100 & 96 & 13 & 92 & 19 \\
\hline Média & 78 & 73 & 82 & & & 85 & 83 & 92 & & & & \\
\hline $\begin{array}{l}\text { Desvio- } \\
\text { Padrão }\end{array}$ & 27 & 32 & 27 & & & 25 & 26 & 17 & & & & \\
\hline $\begin{array}{c}\text { Média } \\
\text { Geral }\end{array}$ & 81 & 77 & 86 & & & 87 & 85 & 94 & & & & \\
\hline $\begin{array}{c}\text { Desvio- } \\
\text { Padrão }\end{array}$ & 27 & 29 & 26 & & & 24 & 25 & 16 & & & & \\
\hline
\end{tabular}


diferentes podem estar revelando muito mais uma dificuldade de integrar características necessárias (cor-posição) para distinguir o alvo a ser alcançado. Mesmo considerando o pequeno número de estímulos a serem memorizados e comparados para se tomar uma decisão correta, a dificuldade pode residir na ausência de uma representação direta da associação (conjunção) das características das letras, das quais a cor faz parte, e não na perda de informação sobre as características individuais de cada estímulo (Walker et al., 1994). Em outras palavras, os resultados deste estudo revelam uma importante distinção entre memória para conjunções de características e memória para itens individuais na memória visual de curto prazo, um fato já constatado em estudos realizados com amostras de adultos (Stefurak \& Boynton, 1986; Treisman, 1977).

Kahneman e Treisman (1984) propuseram um modelo explicativo no qual um arquivo de objeto (object file, uma representação temporária de um objeto na memória de trabalho) manteria as conjunções de características relacionadas às suas coordenadas espaço-temporais. Kahneman, Treisman e Gibbs (1992) argumentam que somente um arquivo de objeto pode ser acessado para uma nova informação e este arquivo normalmente se relaciona com a informação mais recente, o que explica também os efeitos observados em tarefas de memória imediata, tais como as tarefas de recordação livre. A vantagem observada no processamento de estímulos da direita é indicativo da facilidade das crianças dessas faixas etárias pesquisadas em recuperar informação deste arquivo mais recente, e isso independe da idade. No geral, o desempenho melhor das crianças mais velhas pode, com efeito, refletir uma facilidade maior de lidar com a informação viso-espacial (facilitada pela combinação de características necessárias para indicar o que está onde), incluindo aí possíveis mecanismos de recitação da informação visual (Juhel, 1991).

Numa outra perspectiva, Postma (1992, citado por Postma \& De Haan, 1996) argumenta pela existência de dois aspectos distintos que devem ser levados em conta na memória para localização de objetos. Primeiro, realocar um objeto à sua posição espacial inicial requer a recordação das diversas posições que um objeto poderia ocupar. Segundo, o participante deve relembrar quais objetos fora de um determinado conjunto de itens ocuparam qual posição. Para exemplificar a possibilidade de se trabalhar com dois processos separados, Schumann-Hengsteler (1992) mostrou que enquanto as crianças melhoram a recordação de localizações de objetos específicos, não há efeito significativo da idade quando se trata da recordação da localização em si. Walker et al. (1994) obtiveram resultados mostrando que as crianças mais velhas superam as mais novas na tarefa de associação item-posição espacial em que havia três posições seriais possíveis a serem recordadas. Walker et al. (1993) observaram que a memória visual é sensível aos efeitos de posição serial, mas tem características similares em adultos e crianças. Nossos resultados mostram que a recordação da posição espacial das letras apre- sentadas na posição direita foi superior às outras duas posições, mas isso independe da idade. Essa vantagem da posição direita sugere efeitos de processos de aprendizagem da leitura (da esquerda para a direita na nossa cultura) que merecem estudos posteriores. Uma outra interpretação para os efeitos da posição espacial diz respeito aos mecanismos de recitação interna, favorecida pelo desenvolvimento da linguagem e, portanto, da memória verbal.

Esses mecanismos vêm recebendo várias contribuições, uma das quais mostrando que o processo de recitação muda em qualidade com a idade e não só em quantidade. Até 5-6 anos, as crianças usam formas rudimentares de recitação (Gathercole \& Hitch, 1993) que vão, aos poucos, assumindo um caráter de recitação interna mais complexa com o aumento do repertório verbal. Nosso estudo sugere, também, com base na vantagem demonstrada pelos participantes mais velhos (11-12 anos), que estratégias de recitação interna das posições espaciais das letras (considerando que elas podem ser recitadas como esquerda, centro e direita) podem ter sido utilizadas de forma paralela à representação visual (Hitch et al., 1988; Lopes, 1997; Paivio, 1986; Schiano \& Watkins, 1981). O mesmo mecanismo pode ter sido utilizado para as letras. Essa conclusão se deve ao fato de não empregarmos nenhuma estratégia que pudesse impedir o efeito da recitação verbal das letras e das posições espaciais, tais como a supressão articulatória ou o efeito da fala irrelevante. Estudos posteriores deverão empregar delineamentos experimentais para esclarecer essa questão.

\section{Agradecimento}

Agradecemos ao Prof. Osvaldo Freitas de Jesus pelo auxílio na confecção e correção do Abstract.

\section{Referências}

Atkinson,R.C., \& Shiffrin, R. M. (1968). Human memory: a proposed system and its control processes. In K.W. Spence \& T. J. Spence (Orgs.), The psychology of learning and motivation: advances in research and theory (Vol. 2, pp. 89-195). Nova York: Academic.

Baddeley, A . D. (1986). Working memory. Nova York: Oxford University Press.

Baddeley, A . D. (1990). Human memory: theory and practice. Hove, Reino Unido: Lawrence Erlbaum.

Baddeley, A. D. (1992) Is working memory working? The fifteenth Bartlett lecture. Quarterly Journal of Experimental Psychology, 44A, 1-31.

Baddeley, A . D. (1994). Working memory: the interface between memory and cognition. In D. L. Schacter \& E. Tulving (Orgs.), Memory systems 1994. Cambridge: MIT Press.

Baddeley, A . D. (2000). The episodic buffer: a new component of working memory. Trends in cognitive sciences, 4, 417-422.

Baddeley, A. D. (2002). Is working memory still working? European Psychologist, 2, 85-97.

Baddeley.A. D., \& Hitch, G. J. (1974). Working memory. In G. A. Bower (Org.), Recent advances in learning and motivation (pp. 47-90). Nova York: Academic.

Bahrick, A. D., \& Boucher, B. (1968). Retention of visual and verbal code of the same stimuli. Journal of Experimental Psychology, 78, 417-422. 
Brandimonte, M. A., \& Gerbino, W. (1996). When imagery fails: effects of verbal recoding on accessibility of visual memories. In C. Cornoldi et al. (Orgs.), Stretching the imagination: representation and transformation in mental imagery. Nova York: Oxford University Press.

Broadbent, D. E., \& Broadbent, M. H. P. (1981). Recency effects in visual memory. Quarterly_Journal of Experimental Psychology, 33A, 1-15.

Della Sala, S., Gray, C., Baddeley, A., Allamano, N., \& Wilson, L. (1999). Pattern span: a toll for unwelding visuo-spatial memory. Neuropsychologia, 37, 1189-1199.

Flavell, J. H., \& Wellman. H. M. (1977). Metamemory. In R. V. Kail \& J. W. Hagen (Orgs.), Perspectives on the development of memory and cognition (pp. 3-34). Hillsdale: Lawrence Erbaum.

Galera, C., \& Fuhs, C. C. L. (2003). Memória viso-espacial a curto prazo: os efeitos da supressão articulatória e de uma tarefa aritmética. Psicologia: Reflexão e Crítica, 16, 337-348.

Gathercole, S. E., \& Hitch, G. J. (1993). Developmental changes in short-term memory: a revised working memory perspective. In A. F. Collins, S. E. Gathercole, M. A. Conway, \& P. E. Morris (Orgs.), Theories of memory (pp. 189-209). Hove, Reino Unido: Lawrence Erlbaum.

Hecker, R., \& Mapperson, B. (1997). Dissociation of visual and spatial processing in working memory. Neuropsychologia. 35, 599-603.

Hitch, G. J., Halliday, M. S., Dood, A., \& Littler, J. E. (1989). Development of rehearsal in shor-term-memory: differences between pictorial and spoken stimuli. British Journal of Developmental Psychology, 7, 347-362.

Hitch, G. J., Halliday, M. S., \& Littler, J. E. (1989) Item identification time and rehearsal rate as predictors of memory span in children. Quarterly Journal of Experimental Psychology, 41A, 321-337.

Hitch, G. J., Halliday, M. S., Schaafstal,A. M., \& Schraagen, J. M. (1988). Visual working memory in young children. Memory \& Cognition, 16, 120-132.

Hitch, G. J. Woodin, M. E., \& Barker, S. (1989). Visual and phonological components of working memory in children. Memory \& Cognition, 16, 175-185.

Hulme, C., Thomson, N., Muir, C., \& Lawrence, A. (1984). Speech rate and development of short-term memory span. Journal of Experimental Child Psychology, 38, 241-253.

Hulme, C., \& Tordoff, V. (1989). Working memory development: the effects of speech rate, word length, and acoustic similarity on serial recall. Journal of Experimental Child Psychology, 47, 72-87.

Juhel, J. (1991). Spatial abilities and individual differences in visual information processing. Intelligence, 15, 117-137.

Kahneman, D., \& Treisman, A. (1984). Changing views of attention and automaticity. In R. Parasuraman \& J. R. Pomerantz (Orgs.), Varieties of attention. Nova York: Academic.

Kahneman, D., Treisman, A., \& Gibbs, B. J. (1992). The reviewing of object files. Object-specific integration of information. Cognitive Psychology, 24, 175-219.

Kinnear, P. R., \& Gray, C. D. (1995). SPSS for Windows: made simple. Hove, Reino Unido: Erlbaum Taylor \& Francis.

Light, L. L., \& Zelinski, E. M. (1983). Memory for spatial information in young and old adults. Developmental Psychology, 19, 901-906.

Logie, R. H. (1995). Visuo-spatial working memory. Hove, Reino Unido: Lawrence Erlbaum.

Logie, R. H., \& Pearson, D. G. (1997). The inner eye and the inner scribe of visuo-spatial working memory: Evidence from developmental fractionation. European Journal of Cognitive Psychology, 9, 241-257.

Lopes, E. J. (1997). Um estudo experimental sobre a codificação de formas geométricas familiares e não-familiares na memória ativa a curto prazo. Tese de doutorado não-publicada, Universidade de São Paulo, Ribeirão Preto, SP.
Mandler, J. M., Seegmiller, D., \& Day, J. (1977). On the coding of spatial information. Memory \& Cognition, 5, 10-16.

McCormack, P. D. (1982). Coding of spatial information by young and elderly adults. Journal of Gerontology, 1, 80-86.

Milner, D., \& Goodale, M. A. (1995). The visual brain in action. Nova York: Oxford University Press.

Paivio, A . (1986). Mental representations: a dual coding approach. Nova York: Oxford University Press.

Park, C. D., \& James C. Q. (1983). Effect of encoding instrutions on children's spatial and color memory: automaticity. Child Development, 54, 61-68.

Park,C. D., Puglisi, J. T., \& Lutz, R. (1982). Spatial memory in the elderly: effects of intencionality. Journal of Gerontology, 37, 330-335.

Pezdek, K. (1983). Memory for items and their spatial locations by young and elderly adults. Developmental Psychology, 19, 895-900.

Pickering, S. J., Gathercole, S. E., \& Peaker, S. M. (1998). Verbal and visuospatial short-term memory in children: evidence for common and distinct mechanisms. Memory \& Cognition, 26, 1117-1130.

Podgorny, P., \& Garner, W. R. (1979). Reaction time as a measure of inter- and intra-object visual similarity: letters of the alphabet. Perception \& Psychophysics, 26, 37-52.

Postma, A., \& De Haan, E. H. F. (1996). What was where? Memory for object locations. Quarterly Journal of Experimental Psychology, 49A, 178-199.

Quinn, J. G., \& McConnell, J. (1996). Irrelevant picture in visual working memory. Quarterly Journal of Experimental Psychology, 49A, 200-215.

Schiano, D. J., \& Watkins, M. J. (1981). Speech-like coding of pictures in shortterm memory. Memory \& Cognition, 9, 110-114.

Schulmann-Hengsteler, R. (1992). The development of visuo-spatial memory: how to remember location. International Journal of Behavioral Development, 15, 455-471.

Schulmann-Hengsteler, R., Demmel, U., \& Seitz, K. (1992). Visuospatial working memory in children and adults. Memory \& Cognition, 16, 437-445.

Stefurak, D. L., \& Boynton, R. M. (1986). Independence of memory for categorically different colors and shapes. Perception \& Psychophysics, 39, 164-174.

Stoltzfus, E. R., Hasher, L., \& Zacks, R. T. (1996). Working memory and aging: current status of the inhibitory view. In J. T. E. Richardson, R. W. Engle, L. Hasher, R. H. Logie, E. R. Stoltzfus, \& R. T. Zacks (Orgs.), Working memory and human cognition (pp. 66-88). Nova York: Oxford University Press.

Treisman, A . (1977). Focused attention in the perception and retrieval of multidimensional stimuli. Perception \& Psychophysics, 22, 111.

Treisman, A. (1988). Features and objects: the fourteenth Bartlett memorial lecture. Quartely Journal of Experimental Psychology, 40A, 201-237.

Treisman, A., \& Gelade, G. (1980). Feature-integration theory of attention. Cognitive Psychology, 12, 97-137.

Van Ness, F., \& Bouma, H. (1980). On the legibility of segmented numerals. Human Factor, 22, 463-474.

von Wright, J. M., Gebbard, P, \& Karttunem, M. (1975). A developmental study of recall of spatial location. Journal of Experimental Child Psychology, 20, 181-190.

Walker, P., Hitch, G. J., Doyle, A., \& Porter, T. (1994). The development of short-term visual memory in young children. International Journal of Behavioral Development, 17, 73-89.

Walker, P., Hitch, G. J., \& Duroe, S. (1993). The effect of visual similarity on short-term memory for spatial location: implications for the capacity of visual short-term memory. Acta Psychologica, 83, 203-224. 
${ }^{1}$ Os dados desta pesquisa foram apresentados na XXXII Reunião Anual da Sociedade Brasileira de Psicologia, Florianópolis, SC, em outubro de 2002.

Ederaldo José Lopes, doutor em Psicobiologia pela Faculdade de Filosofia, Ciências e Letras de Ribeirão Preto, Universidade de São Paulo, Ribeirão Preto, SP, é professor no Instituto de Psicologia, Universidade Federal de Uberlândia, Uberlândia, MG. Endereço para correspondência: Universidade Federal de Uberlândia; Instituto de Psicologia; Av. Maranhão, s/n, Bairro Umuarama, CEP: 38.405-318. Tel: (34) 3218-2235 e $3218-$ 2296. E-mail: ederaldol@umuarama.ufu.br Renata Ferrarez Fernandes Lopes, doutora em Psicobiologia pela Faculdade de Filosofia, Ciências e Letras de Ribeirão Preto, Universidade de São Paulo, Ribeirão Preto, SP, é professora no Instituto de Psicologia, Universidade Federal de Uberlândia, Uberlândia, MG.

Cesar Alexis Galera, doutor em Psicologia Experimental pelo Instituto de Psicologia da Universidade de São Paulo, SP, é professor no Departamento de Psicologia e Educação, Universidade de São Paulo, Ribeirão Preto, SP. 